\title{
Bayesian compressive sensing for synthetic-aperture radar tomography imaging
}

\author{
${ }^{1}$ Xiaozhen Ren, ${ }^{1}$ Yao Qin and ${ }^{2}$ Lihong Qiao \\ ${ }^{1}$ School of Artificial Intelligence and Big Data, Henan University of Technology, \\ Zhengzhou, 450001, China, rxz235@163.com \\ ${ }^{2}$ Chongqing Key Laboratory of Computational Intelligence, Chongqing University of \\ Posts and Telecommunications, Chongqing, 400065, China
}

Received: 31.07 .2020

\begin{abstract}
To achieve high-resolution three-dimensional images, a number of imaging methods based on compressive sensing (CS) have been suggested in the recent years for synthetic-aperture radar (SAR) tomography. However, the CS-based methods are sensitive to noise. In this work, we develop a new Bayesian compressive sensing (BCS) imaging method for the SAR tomography. In the framework of BCS, a 'sparseness' prior distribution of the imaging scene and an additive noise are properly considered in the imaging process. As a consequence, the BCS-based method under the conditions of low noise levels can provide a better performance than the common norm-based CS methods. The results obtained via simulations of our
\end{abstract} SAR-tomography imaging method confirm its advantages.

Keywords: SAR tomography, Bayesian compressive sensing, three-dimensional imaging, imaging methods

UDC: 621.396 .96

\section{Introduction}

Synthetic-aperture radar (SAR) tomography is a three-dimensional SAR imaging technique, which can provide an estimation of scatterers' distribution along some height direction and generate three-dimensional images of earth surface. The SAR tomography has been receiving a steadily growing interest in the recent years [1-3]. However, this tomography approach requires a synthetic aperture along the height direction, which is often formed by using several multi-pass acquisitions in the same imaging scene. The number of acquisitions is usually very sparse, and their spacing along the height direction is non-uniform. Therefore, the imaging results obtained by these traditional methods are limited due to existence of intense side lobes.

The theory of compressed sensing (CS) developed recently states that it is possible to reconstruct accurately a sparse signal from a small number of random samples $[4,5]$. Motivated by 
the CS theory, efficient methods based on the CS have been suggested for the SAR-tomography imaging. In particular, a CS-based imaging model for the SAR tomography has been worked out in Refs. [6, 7]. It has been shown that the CS-based method manifests better super-resolution properties and point localization accuracies than the traditional methods. In Ref. [8], an iterative reweighted alternating-direction method has been proposed for the SAR-tomography imaging, which reduces computation time notably. A correlated SAR-tomography model has been introduced in the work [9]. It enables one to study the effects of random residual phase and noise. Although the advantages of the CS-based imaging methods for the SAR tomography are obvious, the reconstruction performance degrades rapidly under condition of low signal-to-noise-ratio (SNR).

Recently, a Bayesian compressive-sensing (BCS) framework has been introduced into the SAR-imaging field $[10,11]$. Using the 'sparseness' prior distribution of imaging scene and taking into account the additive noise in the Bayesian formalism, the BCS-based method could provide a better performance than that of the norm-based CS methods, whenever the conditions of low noise levels hold true $[12,13]$. However, the 'sparseness' prior distributions used in Refs. [10, 11] are nothing but generalized Gaussian and Laplace random models, which represent in fact invalid low-dimensional models [14]. A Cauchy prior distribution has been suggested in Ref. [15] for the case of four-dimensional SAR imaging, although the imaging result remains related to the scale parameter of the Cauchy distribution. Inspired by the 'hierarchical prior' approach used in Ref. [16], below we investigate a new SAR-tomography imaging method based on the BCS. It enables improving the performance of the CS-based SAR-tomography imaging at low noise levels.

The article is organized as follows. The principles of SAR-tomography imaging are described in Section 2. Section 3 introduces a scheme of the BCS-based SAR-tomography imaging. The performance of our method in relation to the noise-suppression properties is presented in Section 4. Finally, Section 5 summarizes the our main results.

\section{SAR-tomography imaging principles}

A typical geometry of the SAR-tomography imaging is shown in Fig. 1. The SAR sensors pass along $M$ different orbital positions. For the sake of simplicity, the orbits are supposed to be parallel. The central orbital position is defined as a reference position with the altitude $H$ and its incidence angle is equal to $\theta$. The parameter $\varepsilon$ denotes the angle between the baseline and the horizontal line. The axes $x, y, r$ and $z$ denote respectively the range, the azimuth, the slant range and the altitude directions. The height direction $s$ is defined as a direction perpendicular to both the azimuth and the slant range. For any target with the azimuth $y^{\prime}$, the slant range $r$ ' and the heighs $s$, we 
record its positions' distribution as $\left(y^{\prime}, r^{\prime}, s^{\prime}\right)$. After co-detecting and phase-calibrating $M$ SAR images, the data received for a fixed azimuth-slant range cell $\left(y_{i}, r_{i}\right)$ can be written as [17]

$$
y_{m}=\int \gamma(s) g_{m}(s) d s
$$

where

$$
g_{m}(s)=\exp \left[j 2 \pi\left(\frac{2 s}{\lambda R} b_{\perp m}\right)\right], \quad m=1,2, \cdots, M .
$$

Here $\gamma(s)$ represents the complex reflectivity function, $\lambda$ the radar wavelength, $\mathrm{R}$ the range between the reference baseline and the scattering centre, and $b_{\perp m}$ the height baseline.

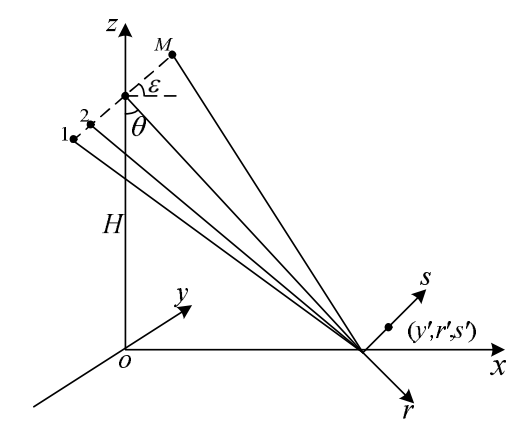

Fig. 1. Geometry of SAR-tomography imaging.

For the numerical analysis, the continuous signal model for the SAR-tomography imaging given by Eq. (1) can be expressed in a matrix form as follows:

$$
\boldsymbol{y}=\boldsymbol{\Phi}+\boldsymbol{n},
$$

where $\boldsymbol{y}$ is an $M \times 1$ measurement vector, of which components $y_{m} \quad(m=1,2, \cdots M)$ are the data points received with an SAR-tomography system. Note that $v$ implies an $N \times 1$ signal vector, which is stacked from $\gamma(s)$ samples. Finally, $\boldsymbol{n}$ denotes the complex Gaussian vector with the zero mean and the power $\sigma^{2}$, and $\Phi$ is the $M \times N$ sensing matrix. Its components $\phi_{m n}$ can be obtained from Eq. (2) and expressed as

$$
\phi_{m n}=\exp \left(j 2 \pi w_{m} s_{n}\right)
$$

with $w_{m}=2 b_{\perp m} /(\lambda R)$. In other words, the aim of the SAR-tomography imaging is to retrieve the reflectivity coefficient $v$.

\section{Bayesian compressive sensing for tomography SAR imaging}

Below we employ the BCS-based method to estimate the reflectivity coefficient $\boldsymbol{v}$ of the SAR 
tomography. In the framework of Bayesian formalism, the compressibility of signal is realized by placing the sparseness prior on it. The sparseness priors used commonly are the generalized Gaussian and Laplace density functions. However, both of those random models in fact represent invalid low-dimensional models (see Ref. [14]). In the present work, we employ the hierarchical prior as the sparseness prior of the reflectivity coefficient $\boldsymbol{v}$. It has been already proven as efficient in obtaining the sparse solution to the regression and classification tasks [18].

First, we define a zero-mean Gaussian prior distribution for the reflectivity coefficient $v$ :

$$
p(v \mid \alpha)=\prod_{i=1}^{N} N\left(v_{i} \mid 0, \alpha_{i}^{-1}\right)
$$

where $\boldsymbol{\alpha}$ is a vector of $N$ hyper-parameters and $N(\cdot)$ denotes the Gaussian distribution. Then, to complete specification of this hierarchical prior, we consider the Gamma distribution over $\alpha$ and the inverse of the noise variance, $\beta=1 / \sigma^{2}$. This yields

$$
\begin{gathered}
p(\alpha \mid a, b)=\prod_{i=1}^{N} \operatorname{Gamma}\left(\alpha_{i} \mid a, b\right), \\
p(\beta \mid c, d)=\operatorname{Gamma}(\beta \mid c, d),
\end{gathered}
$$

where

$$
\begin{gathered}
\operatorname{Gamma}(\alpha \mid a, b)=\Gamma(a)^{-1} b^{a} \alpha^{a-1} e^{-b \alpha}, \\
\Gamma(a)=\int_{0}^{\infty} t^{a-1} e^{-t} d t .
\end{gathered}
$$

To make these priors non-informative, the parameters $a, b, c$ and $d$ should be fixed at small values. It has been proven that the uniform hyper-priors can be obtained by setting these parameters to zero [18]. Therefore, the parameters $a, b, c$ and $d$ are set to zero further on.

Generally, the components of $\boldsymbol{n}$ are approximated as a zero-mean complex Gaussian noise. Therefore, the likelihood function of the observation data $y$ reads as

$$
p\left(\boldsymbol{y} \mid \boldsymbol{v}, \sigma^{2}\right)=\left(\frac{1}{2 \pi \sigma^{2}}\right)^{M} \exp \left\{-\frac{\|\boldsymbol{y}-\boldsymbol{\Phi} \boldsymbol{v}\|_{2}^{2}}{2 \sigma^{2}}\right\} .
$$

From the Bayes rule, the posterior distribution of the reflectivity coefficient $v$ can be obtained as

$$
p(\boldsymbol{v} \mid \boldsymbol{y}, \boldsymbol{\alpha}, \beta)=N(\boldsymbol{v} \mid \boldsymbol{\mu}, \boldsymbol{\Sigma}) .
$$

Here the posterior covariance $\boldsymbol{\Sigma}$ and the mean $\boldsymbol{\mu}$ are given by

$$
\Sigma=\left(\beta \boldsymbol{\Phi}^{\mathrm{T}} \boldsymbol{\Phi}+\mathbf{A}\right)^{-1},
$$




$$
\boldsymbol{\mu}=\beta \Sigma \Phi^{\mathrm{T}} \boldsymbol{y}
$$

with $\mathbf{A}=\operatorname{diag}\left(\alpha_{1}, \alpha_{2}, \cdots, \alpha_{N}\right)$.

Once the parameters $\alpha$ and $\beta$ are given, the maximum posterior estimate of the reflectivity coefficient $v$ can be obtained:

$$
\hat{\boldsymbol{v}}=\boldsymbol{\mu}=\beta \boldsymbol{\Sigma} \boldsymbol{\Phi}^{\mathrm{T}} \boldsymbol{y} .
$$

From Eq. (11), the marginal likelihood for $\alpha$ and $\beta$ can be written as

$$
L(\boldsymbol{\alpha}, \beta)=-\frac{1}{2}\left(N \log 2 \pi+\log \left|\sigma^{2} \mathbf{I}+\boldsymbol{\Phi} \mathbf{A}^{-1} \boldsymbol{\Phi}^{\mathrm{T}}\right|+\boldsymbol{y}^{\mathrm{T}}\left(\sigma^{2} \mathbf{I}+\boldsymbol{\Phi} \mathbf{A}^{-1} \boldsymbol{\Phi}^{\mathrm{T}}\right)^{-1} \boldsymbol{y}\right),
$$

where I denotes the identity matrix.

Using the standard expectation-maximization method [19], one can obtain the estimation for $\alpha$ and $\beta$ :

$$
\begin{gathered}
\alpha_{i}^{\text {new }}=\frac{\gamma_{i}}{\mu_{i}^{2}}, \\
\beta^{\text {new }}=\frac{N-\sum_{i} \gamma_{i}}{\|\boldsymbol{y}-\boldsymbol{\Phi} \boldsymbol{\mu}\|^{2}},
\end{gathered}
$$

where $\mu_{i}$ is the $i$-th element of the posterior mean $\mu$ and the parameter $\gamma_{i}$ is defined by

$$
\gamma_{i}=1-\alpha_{i} \Sigma_{i i}
$$

with $\Sigma_{i i}$ being the $i$-th diagonal element of the posterior covariance $\Sigma$.

Following from the analysis performed above, the parameters $\alpha$ and $\beta$ can be updated from Eqs. (16) and (17), concurrent with updating of the posterior covariance $\Sigma$ and the mean $\mu$ from Eqs. (12) and (13). This leads to an iterative algorithm, which goes on until the convergence criteria are satisfied. When all the azimuth-slant range cells of a scene have been processed according to the above steps, a three-dimensional SAR-tomography image can readily be obtained.

\section{Experiments}

To analyze the performance of our method suggested for the SAR-tomography imaging, we perform the following experiments using simulated data. Let the height of the centre of SAR platform be equal to $5000 \mathrm{~m}$, the incidence angle to $45^{\circ}$ and the wavelength of the SAR system to $0.23 \mathrm{~m}$. In the simulation that follows, the observed scene is composed of two cube buildings, and the echo signal acquired by radar comes from the roofs of the two buildings only. Fig. 2 shows the positions of the two buildings, which are parallel to the azimuth direction. 
Assume we have acquired the SAR-tomography signals from 11 multiple passes, and the de-correlation effect is introduced by adding the Gaussian noise. We compare the reconstruction results obtained using our method with those of the standard CS method based upon orthogonal matching pursuit (OMP) [20] and the method of weighted compressive sensing (WCS) [21]. We adopt the reconstruction error $\|\hat{x}-x\|_{2} /\|x\|_{2}$ as the index evaluating the reconstruction quality, with $\hat{x}$ and $x$ being defined as the estimated and true images, respectively.

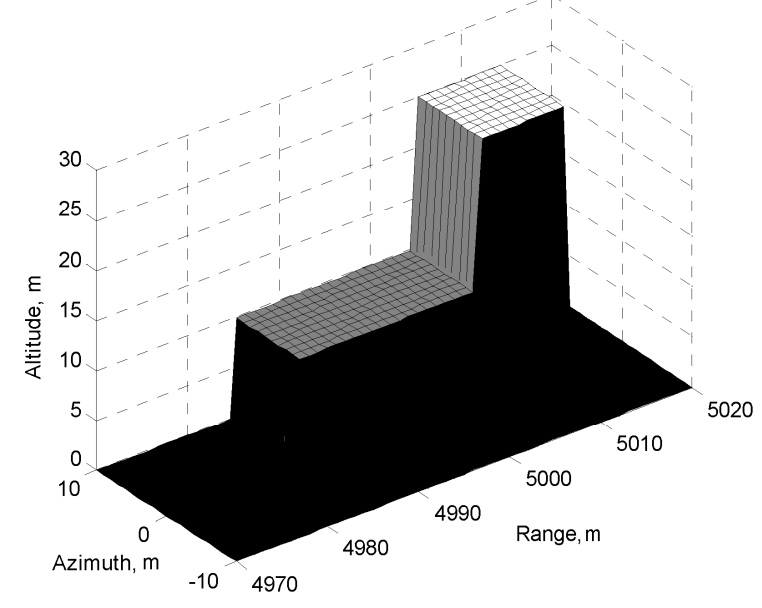

Fig. 2. Imaging scene to be reconstructed (see the text).

In order to check the viability of the method suggested in the present study, we select a slice along the azimuth and height directions corresponding to the slant range position $7050 \mathrm{~m}$. Fig. 3 compares the results obtained using the OMP, the WCS and our method. They refer to the selected azimuth-height slice under condition when the SNR is equal to $5 \mathrm{~dB}$. From Fig. $3 \mathrm{a}$, it is clear that the OMP method is sensitive to the noise. There are many artefacts and some of the targets are detected at wrong positions. From Fig. 3b and Fig. 3c it can be seen that the WCS and the method suggested by us result in better imaging results, if compared with the OMP. However, our method manifests a better performance than the WCS. The targets are detected at right positions by our method, and no artefacts appear at all. As a conclusion, the method described in the present work outperforms the other two methods under noisy conditions.

To illustrate further the advantages of our method, we extract for the analysis the azimuth-slant range resolution cell that corresponds to the coordinates $(0,7052)$. Panels a-c in Fig. 4 show the reconstruction results for the height reflectivity profile obtained using the OMP and WCS methods and our method. Here the SNR equal to $10 \mathrm{~dB}$ is taken. One can see that the reconstructions of the height reflectivity profile formed by all of the three methods exhibit good performance in the case of high SNR, $10 \mathrm{~dB}$. 


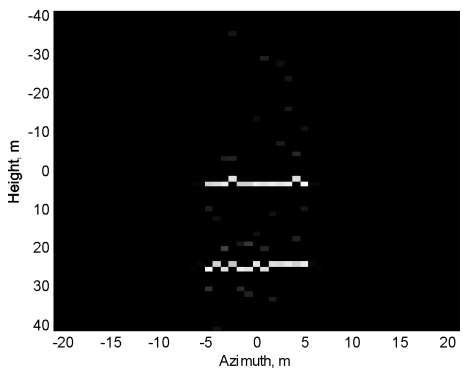

(a)

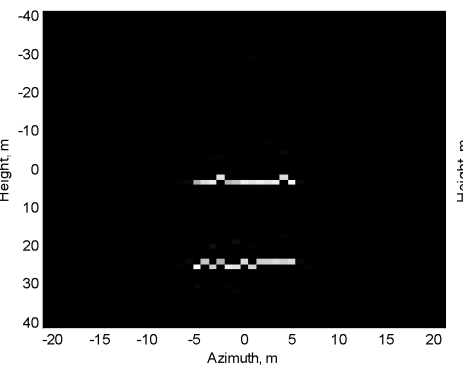

(b)

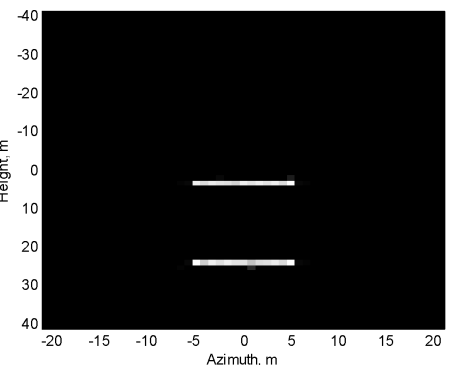

(c)

Fig. 3. Comparison of reconstruction results obtained using OMP (a), WCS (b) and our method (c) under the same SNR value equal to $5 \mathrm{~dB}$.

Fig. $4 \mathrm{~d}$ shows the reconstruction of the height reflectivity profile performed with the OMP method for the case of $\mathrm{SNR}=5 \mathrm{~dB}$. Now it is evident that the reconstruction quality of the OMP method decays significantly with decreasing SNR, while the artificial scattering centres are serious enough. Fig. 4e and Fig. 4f compare the reconstruction results obtained for the height reflectivity profile with the WCS method and the method suggested by us. Here the same case of SNR $=5 \mathrm{~dB}$ is analyzed. The data proves that the both methods are robust to noise, although our method provides much clearer image under noisy conditions.

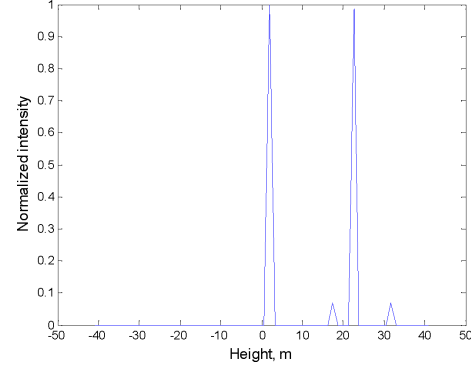

(a)

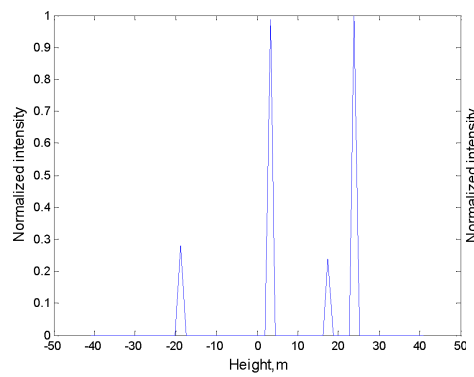

(d)

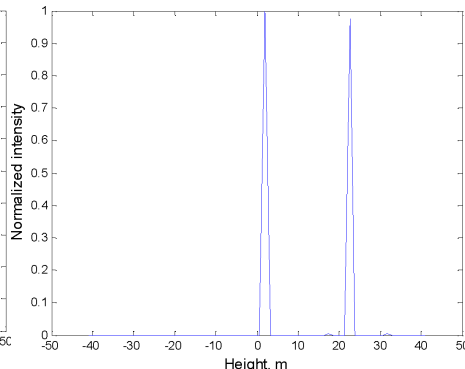

(b)

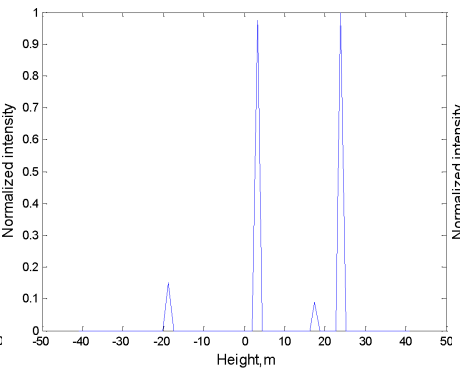

(e)

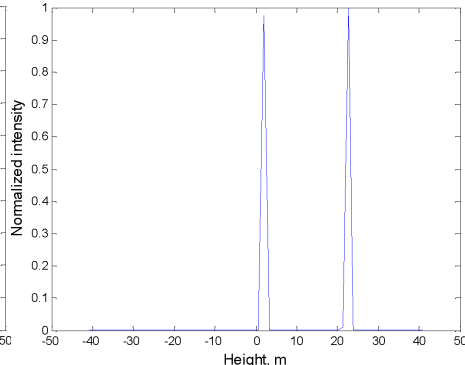

(c)

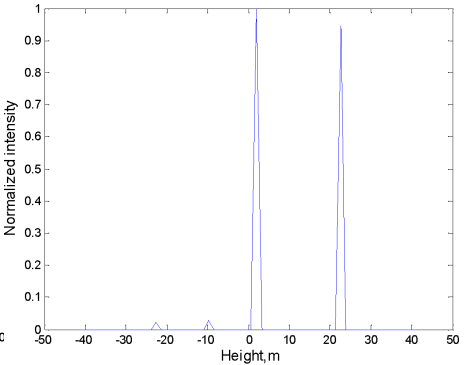

(f)

Fig. 4. Comparison of reconstruction results for the elevation reflectively profile, which are obtained using OMP $(a, d)$, WCS (b, e) and our method (c, f) at different noise levels: SNR $=10 d B(a, b, c)$ and SNR $=5 d B(d, e, f)$. 
Finally, we have calculated the reconstruction errors to evaluate the performance of the OMP, the WCS and our method. Fig. 5 shows the reconstruction error as a function of SNR. It is natural that the reconstruction error for all the methods is very low at the SNRs greater than $10 \mathrm{~dB}$. However, the performance of the OMP degrades significantly when the SNR decreases. Our method provides a better performance than the OMP and the WCS and remains more robust to the noise.

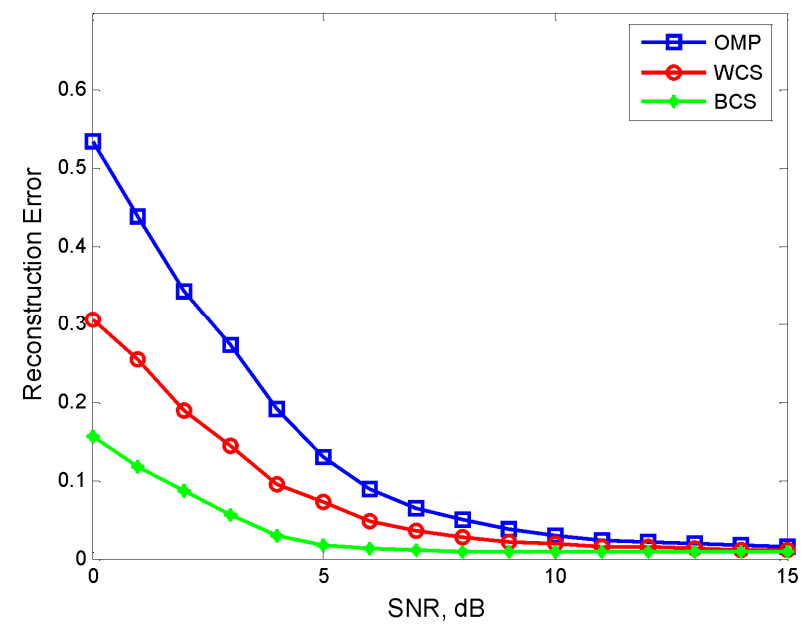

Fig. 5. Reconstruction errors calculated for different methods (see the legend) versus SNR level.

\section{Conclusion}

In this work we have suggested a new method for the SAR-tomography imaging, which is based on the BCS approach. The most noticeable advantage of our method is that the hierarchical prior and the additive noise are simultaneously considered within the process of SAR-tomography imaging. As a consequence, the BCS-based method provides a better performance than that typical for the norm-based CS methods, whenever the noise levels remain low enough.

The numerical results derived by us using the simulation data testify that the imaging performance revealed by our method is better than that obtained using the OMP and WCS methods, especially under the conditions of low SNRs.

\section{Acknowledgments}

This work was supported by the National Natural Science Foundation of China (under the Grant U1904120), the Henan Science and Technology Department Science and Technology Research Program (under the Grants 182102310759 and 182300410146), and the Fundamental Research Funds for the Henan Provincial Colleges and Universities (under the Grants 2018RCJH09 and 2017RCJH01). 


\section{References}

1. Reigber A, Lombardini F, Viviani F, Nannini M and Martinez A, 2015. Three-dimensional and higher-order imaging with tomographic SAR: techniques, applications, issues. Proc. IGARSS. 2015: 2915-2918.

2. Lombardini $\mathrm{F}$ and Tebaldini S, 2017. Multidimensional SAR tomography: methods and applications. Proc. IGARSS. 2017: 2460-2463.

3. Aghababaee $\mathrm{H}$ and Sahebi M R, 2018. Model-based target scattering decomposition of polarimetric SAR tomography. IEEE Trans. Geosci. Remote Sens. 56: 972-983.

4. Candes E J, Romberg J and Tao T, 2006. Robust uncertainty principles: Exact signal reconstruction from highly incomplete frequency information. IEEE Trans. Inform. Theory. 52: 489-509.

5. Donoho D, 2006. Compressed sensing. IEEE Trans. Inform. Theory. 52: 1289-1306.

6. Zhu X and Bamler R, 2010. Tomography SAR inversion by L1-norm regularization - the compressive sensing approach. IEEE Trans. Geosci. Remote Sens. 48: 3839-3846.

7. Zhu X and Bamler R, 2014. Superresolving SAR tomography for multidimensional imaging of urban areas: compressive sensing-based TomoSAR inversion. IEEE Signal Process. Mag. 31: $51-58$.

8. Wang $\mathrm{X}, \mathrm{Xu} \mathrm{F}$ and Jin $\mathrm{Y}$ Q, 2017. The iterative reweighted alternating direction method of multipliers for separating structural layovers in SAR tomography. IEEE Geosci. Remote Sens. Lett. 14: $1883-1887$.

9. Bo Yang, Huaping Xu, Wei Liu, Yanan You and Xiaozhen Xie, 2018. Realistic lower bound on elevation estimation for tomographic SAR. IEEE J. Sel. Top. App. Earth Obs. Remote Sens. 11: 2429-2439.

10. Jungang Yang, Tian Jin, Chao Xiao and Xiaotao Huang, 2019. Compressed sensing radar imaging: fundamentals, challenges, and advances. Sensors. 19: 3100.

11. Ren X Z, Chen L N and Yang J, 2014. 3D imaging algorithm for down-looking MIMO array SAR based on Bayesian compressive sensing. Int. J. Anten. Propag. 2014: 612326.

12. Ji S, Xue Y and Carin L, 2008. Bayesian compressive sensing. IEEE Trans. Signal Process. 56: 2346-2356.

13. Zhang X Z, Qin J H and Li G J, 2013. SAR target classification using Bayesian compressive sensing with scattering centers features. Prog. Electromagn. Res. 136: 385-407.

14. Baraniuk R G, Cevher V and Wakin M B, 2010. Low-dimensional models for dimensionality reduction and signal recovery: a geometric perspective. Proc. IEEE. 98: 959-971.

15. Ren X Z and Chen L N, 2014. Four-dimensional SAR imaging algorithm using Bayesian compressive sensing. J. Electromagnet. Waves and Appl. 28: 1661-1676.

16. $\mathrm{Xu} \mathrm{J}, \mathrm{Pi} \mathrm{Y}$ and $\mathrm{Cao} \mathrm{Z}, 2012$. Bayesian compressive sensing in synthetic aperture radar imaging. IET Radar Sonar Navig. 6: 2-8.

17. Fornaro G, Serafino F and Soldovieri F, 2003. Three-dimensional focusing with multipass SAR data. IEEE Trans. Geosci. Remote Sens. 41: 507-517. 
18. Tipping M E, 2001. Sparse Bayesian learning and the relevance vector machine. J. Mach. Learn. Res. 1: 211-244.

19. Zhang Z and Rao B D, 2011. Sparse signal recovery with temporally correlated source vectors using sparse Bayesian learning. IEEE J. Sel. Top. Signal Process. 5: 912-926.

20. He S, Pang L, Zhang X D, Liu H, Bi H, Ai L P, Sun M X and Wang Y, 2016. SAR tomography imaging based on generalized orthogonal matching pursuit - the case study of Pangu 7 star hotel in Beijing. Proc. IGARSS 2016: 6665-6668.

21. Hyungkeuk Lee and Hyun-Woo Lee, 2014. A weighted compressive sensing method for multi-view images. Proc. Inform. and Commun. Technol. Convergence (ICTC), 2014: 867-869.

Ren X., Qin Y. and Qiao L. 2020. Bayesian compressive sensing for synthetic-aperture radar tomography imaging. Ukr.J.Phys.Opt. 21: 191 - 200. doi: 10.3116/16091833/21/4/191/2020

Анотація. Для одержання тривимірних зображень із високою роздільною здатністю в останні роки запропоновано низку заснованих на компресійному зондуванні (КЗ) методів візуалізаиії для радіолокаційної томографії із синтетичною апертурою (РТСА). Однак методи на основі КЗ чутливі до шуму. В иій роботі розроблено новий метод візуалізації для РТСА на основі байєсівського компресійного зондування (БКЗ). У процесі візуалізації в рамках БКЗ належно враховують «розрідженість» попереднього розподілу сиени зображення та додатковий шум. Як наслідок, за умов низьких рівнів шумів метод, заснований на БКС, забезпечує кращі показники, порівняно із загальноприйнятими методами КС на основі норм. Для перевірки намого методу візуалізації в рамках РТСА-томографії проведено експерименти з отриманими моделюванням даними, які підтвердили переваги иьвого методу. 